\title{
Supplementary Zeeman Data for the First Spectrum of Ruthenium (RuI)
}

\author{
J. Rand McNally, Jr. ${ }^{1}$ and K. G. Kessler
}

(July 22, 1959)

\begin{abstract}
Zeeman data are listed for 207 lines of $\mathrm{Ru}$ I between 2400 and $5400 \mathrm{~A}$, all of which have been classified. The spectrograms were made at the Massachusetts Institute of Technology and were analyzed there and at the National Bureau of Standards.
\end{abstract}

Harrison and McNally ${ }^{2}$ published Zeeman data for 450 lines of the first spectrum of ruthenium $(\mathrm{Ru} \mathrm{I})$ in $1940 . \quad$ In addition unresolved patterns were measured by McNally for 175 lines, but these have never been published. ${ }^{3}$ Further Zeeman observations were made in 1949 by Meggers with the Bitter magnet at the Massachusetts Institute of Technology. These plates were measured by Kessler in the course of preparing material for the preceding paper. Zeeman data for 32 additional lines obtained from this last set of observations, together with the unpublished material from McNally's thesis for 175 lines are listed below.

The observations were all made with electrodes of 1 part ruthenium powder mixed with 5 parts of silver powder. The experimental conditions and techniques used are fully described by Harrison and MeNally. ${ }^{2}$

The wavelengths (in air) of these lines are given in column 1 of table 1 . The observed $g$-value for the lower energy level involved in the transition producing the line is given in column 2 , and that for the upper level is given in column 3 . The complete designations for these lines are given in the preceding paper.

In the case of unresolved patterns of classified lines, where a $g$-value is known for one of the energy levels, the other $g$-value can be calculated from the separation of the strongest " $n$ " components when the $J$-values are unequa!, or from the separation of the " $p$ " components when the $J$-values are equal. The $g$-values derived in this way are designated " $n$ " or " $p$ " to indicate which set of components was used. The known observed $g$-value that was used in the calculation is shown in parentheses.

The entry "Mc" in column 4 indicates that the data are taken from McNally's thesis, ${ }^{3}$ and " $\mathrm{K}$ " denotes $g$-values determined at NBS.

1 Oak Ridge National Laboratory.

2 G. R. Harrison and J. R. MeNally, Jr. Phys, Rev. 58, 703 (1940).

3 J. R. MeNally, Jr., Thesis, Mass. Inst. Technol, (1941) unpublished.
TABLE 1.

\begin{tabular}{|c|c|c|c|c|}
\hline Wavelength & Lower level & \multicolumn{2}{|c|}{ Upper level } & Source \\
\hline$A$ & Obs. $g$ & Obs. g & & \\
\hline 2392. 425 & 0.005 & 0.388 & & $\mathrm{Mc}$ \\
\hline 2464.699 & 1. 49 & 2. 02 & & K \\
\hline 2467.576 & 1. 21 & 1. 21 & & $\mathrm{~K}$ \\
\hline 2476. 869 & 1. 532 & 1. 255 & & $\mathrm{~K}$ \\
\hline 2496.56 & 1. 429 & 1. 134 & & $\mathrm{~K}$ \\
\hline 2501.885 & (1. 089) & 1. 029 & $n$ & $\mathrm{Mc}$ \\
\hline 2544.22 & 1. 31 & 1. 31 & & $\mathrm{~K}$ \\
\hline 2558.540 & (1. 196) & 1. 24 & $n$ & $\mathrm{~K}$ \\
\hline 2560.265 & (1. 624) & 1. 322 & $p$ & $\mathrm{Mc}$ \\
\hline 2567.893 & $(1.284)$ & 1. 541 & $p$ & $\mathrm{Mc}$ \\
\hline 2592. 022 & 1. 624 & 1. 372 & & $\mathrm{~K}$ \\
\hline 2593.700 & 1. 033 & 1. 092 & & $\mathrm{Mc}$ \\
\hline 2605.347 & 1. 428 & 1. 022 & & $\mathrm{~K}$ \\
\hline 2605. 853 & 1. 07 & 1. 06 & & $\mathrm{~K}$ \\
\hline 2611. 045 & 1. 22 & 1. 62 & & $\mathrm{~K}$ \\
\hline 2651. 839 & 1. 267 & 1. 485 & & $\mathrm{~K}$ \\
\hline 2702.833 & 1. 183 & 1. 454 & & $\mathrm{~K}$ \\
\hline 2721.562 & 1. 248 & 1. 473 & & $\mathrm{~K}$ \\
\hline 2730.932 & 1. 066 & 1. 470 & & $\mathrm{~K}$ \\
\hline 2735.727 & $(1.397)$ & 1. 474 & $n$ & $\mathrm{Mc}$ \\
\hline 2754.603 & (1. 089) & 1. 552 & $p$ & $\mathrm{Mc}$ \\
\hline 2810.029 & 1. 269 & 1. 462 & & $\mathrm{Mc}$ \\
\hline 2817. 092 & 0. 998 & 1. 560 & & $\mathrm{~K}$ \\
\hline 2840.537 & (1. 284) & 1. 198 & $p$ & $\mathrm{Mc}$ \\
\hline 2891.645 & (1. 16) & 1. 16 & $n$ & $\mathrm{Mc}$ \\
\hline 2913. 163 & 1. 71 & $0 / 0$ & $n$ & $\mathrm{Me}$ \\
\hline 2914. 294 & $0 / 0$ & 1. 115 & & $\mathrm{Mc}$ \\
\hline 2915. 614 & (1. 190) & 1. 030 & $p$ & $\mathrm{Mc}$ \\
\hline 2916. 251 & $(1.35)$ & 1. 35 & $n$ & $\mathrm{Mc}$ \\
\hline 2917.132 & (1. 684) & 1. 115 & $p$ & $\mathrm{Mc}$ \\
\hline 2920.949 & $0 / 0$ & 0.440 & & $\mathrm{~K}$ \\
\hline 2928. 487 & (1. 684) & 1. 084 & $p$ & $\mathrm{Mc}$ \\
\hline 2936. 005 & (1. 232) & 1. 527 & $p$ & $\mathrm{Mc}$ \\
\hline 2937. 336 & (1. 795) & 1. 533 & $p$ & $\mathrm{Mc}$ \\
\hline 2939. 676 & $(1.086)$ & 0.923 & $p$ & $\mathrm{Mc}$ \\
\hline 2950.532 & (1. 041) & 1. 169 & $p$ & $\mathrm{Me}$ \\
\hline 2955. 348 & $(0.757)$ & 1. 163 & $p$ & $\mathrm{Me}$ \\
\hline 2993. 273 & 1. 218 & 1. 298 & & $\mathrm{Mc}$ \\
\hline 3008.797 & $(1.25)$ & 1. 25 & $n$ & $\mathrm{Mc}$ \\
\hline 3013. 354 & (1. 196) & 1. 234 & $n$ & \\
\hline
\end{tabular}


Table 1.-Continued

\begin{tabular}{|c|}
\hline Wavelength \\
\hline$A$ \\
\hline $\begin{array}{l}3057.353 \\
3064.834 \\
3077.542 \\
3084.521 \\
3096.565\end{array}$ \\
\hline $\begin{array}{l}3105.278 \\
3118.065 \\
3129.835 \\
3132.874 \\
3144.265\end{array}$ \\
\hline $\begin{array}{l}3153.831 \\
3170.088 \\
3174.128 \\
3179.025 \\
3193.509\end{array}$ \\
\hline $\begin{array}{l}3250.002 \\
3251.893 \\
3277.564 \\
3306.179 \\
3324.999\end{array}$ \\
\hline $\begin{array}{l}3341.090 \\
3345.316 \\
3348.704 \\
3351.930 \\
3356.201\end{array}$ \\
\hline $\begin{array}{l}3364.100 \\
3378.034 \\
3385.161 \\
3390.899 \\
3414.641\end{array}$ \\
\hline $\begin{array}{l}3428.319 \\
3443.153 \\
3446.670 \\
3455.385 \\
3456.621\end{array}$ \\
\hline $\begin{array}{l}3459.585 \\
3467.051 \\
3494.254 \\
3498.944 \\
3502.418\end{array}$ \\
\hline $\begin{array}{l}3537.941 \\
3587.204 \\
3601.487 \\
3625.197 \\
3626.740\end{array}$ \\
\hline $\begin{array}{l}3672.059 \\
3672.378 \\
3676.952 \\
3701.312 \\
3738.914\end{array}$ \\
\hline $\begin{array}{l}3742.798 \\
3746.218 \\
3781.171 \\
3786.065 \\
3803.191\end{array}$ \\
\hline
\end{tabular}

Table 1.-Continued

\begin{tabular}{|c|c|c|c|c|}
\hline Wavelength & Lower level & \multicolumn{2}{|c|}{ Upper level } & Source \\
\hline$A$ & Obs. g & Obs. $g$ & & \\
\hline 3814.848 & 1. 14 & 1. 21 & & Me \\
\hline 3817. 293 & (1. 164) & 1. 189 & $n$ & $\mathrm{Mc}$ \\
\hline 3835.983 & 1. 186 & 1. 540 & & $\mathrm{Mc}$ \\
\hline 3838. 069 & (1. 563$)$ & 1. 526 & $n$ & $\mathrm{Me}$ \\
\hline 3850.441 & 1. 45 & 1. 45 & $n$ & $\mathrm{Mc}$ \\
\hline 3857.551 & $(0.992)$ & 1. 012 & $n$ & $\mathrm{Mc}$ \\
\hline 3862.690 & $(0.834)$ & 0.853 & $n$ & $\mathrm{Me}$ \\
\hline 3864.851 & $0 / 0$ & 0.78 & $n$ & $\mathrm{Mc}$ \\
\hline 3905. 993 & $(1.343)$ & 1. 061 & $p$ & Mc \\
\hline 3923. 486 & 1. 033 & 1. 048 & & $\mathrm{M}_{s}$ \\
\hline 3924. 636 & $(1.007)$ & 1. 07 & $p$ & K \\
\hline 3937. 919 & 1. 53 & 1. 48 & & $\mathrm{Mc}$ \\
\hline 3941.672 & (1.315) & 1. 711 & $p$ & $\mathrm{Mc}$ \\
\hline 3950.041 & $(1.007)$ & 0.962 & $n$ & $\mathrm{Mc}$ \\
\hline 3952. 290 & $(0.676)$ & 1. 006 & & $\mathrm{Mc}$ \\
\hline 3974.504 & (1. 420) & 1. 470 & & $\mathrm{Mc}$ \\
\hline 4005. 089 & (1.175) & 1. 004 & $p$ & $\mathrm{Mc}$ \\
\hline 4014. 153 & 0.69 & 0.69 & & $\mathrm{~K}$ \\
\hline 4026.492 & $(1.070)$ & 0.703 & & $\mathrm{~K}$ \\
\hline 4028. 434 & $(1.086)$ & 1. 358 & $p$ & $\mathrm{Mc}$ \\
\hline 4040. 474 & 0.997 & 1. 055 & & K \\
\hline 4046.883 & 0.693 & 1. 058 & & K \\
\hline 4062.854 & $(1.007)$ & 0.926 & $p$ & $\mathrm{Me}$ \\
\hline 4076. 730 & 1. 233 & 0.524 & $P$ & $\mathrm{~K}$ \\
\hline 4108. 055 & $(1.190)$ & 1. 487 & $p$ & $\mathrm{Mc}$ \\
\hline 4134. 854 & (1.684) & 1. 066 & $p$ & $\mathrm{Mc}$ \\
\hline 4156. 254 & $(1.007)$ & 1. 035 & $p$ & $\mathrm{Me}$ \\
\hline 4159. 168 & (1.041) & 1. 036 & $n$ & $\mathrm{Mc}$ \\
\hline 4175.436 & $(1.007)$ & 1. 219 & $p$ & $\mathrm{Mc}$ \\
\hline 4182.455 & $(1.175)$ & 1. 137 & $n$ & $\mathrm{Mc}$ \\
\hline 4185. 465 & 1. 08 & 1. 08 & & K \\
\hline 4239. 660 & $(0.697)$ & 1. 081 & $n$ & K \\
\hline 4281.941 & 1. 164 & 1. 174 & & $\mathrm{Mc}$ \\
\hline 4312.494 & 0.71 & $0 / 0$ & $p$ & $\mathrm{Mc}$ \\
\hline 4314.308 & 1. 590 & 1. 609 & & $\mathrm{~K}$ \\
\hline 4325.059 & 1. 327 & 1. 438 & & K \\
\hline 4338. 675 & (1. 343$)$ & 0.938 & $p$ & $\mathrm{Me}$ \\
\hline 4340.351 & (1.041) & 0.895 & $p$ & $\mathrm{Mc}$ \\
\hline 4364.108 & (1. 343$)$ & 0.796 & $p$ & $\mathrm{Mc}$ \\
\hline 4394.970 & (1. 164) & 1. 047 & $p$ & $\mathrm{Mc}$ \\
\hline 4438. 343 & $(1.162)$ & 0.438 & $p$ & $\mathrm{~K}$ \\
\hline 4439. 745 & $(1.086)$ & 1. 024 & $n$ & $\mathrm{Mc}$ \\
\hline $4645.09-$ & 1. 005 & (1.013) & & $\mathrm{Mc}$ \\
\hline 4774.004 & $(0.927)$ & 0.900 & $n$ & $\mathrm{Mc}$ \\
\hline 5133.895 & $(0.757)$ & 1. 029 & $p$ & $\mathrm{Mc}$ \\
\hline 5142.772 & (1. 420) & 1.370 & $n$ & $\mathrm{Mc}$ \\
\hline 5171.026 & (1. 447$)$ & 1. 396 & $n$ & $\mathrm{Mc}$ \\
\hline 5266. 469 & 1. 337 & 1.595 & & $\mathrm{Mc}$ \\
\hline 5280.812 & 1. 426 & 1.426 & $n$ & $\mathrm{Mc}$ \\
\hline 5284.089 & 1. 282 & 1. 632 & & $\mathrm{Mc}$ \\
\hline 5377.840 & 1. 22 & 1. 19 & & $\mathrm{Mc}$ \\
\hline
\end{tabular}

Washington, D.C.

(Paper 63A3-18) 